\title{
Asymptomatic entrapped denture in the hypopharynx
}

\author{
Shogo Hanai ${ }^{1}$, Teruhiko Terasawa ${ }^{1}$, and Mitsunaga Iwata ${ }^{1}$ \\ ${ }^{1}$ Fujita Health University
}

January 21, 2021

\begin{abstract}
A 71-year-old asymptomatic man was brought to our emergency department for ingesting a removable partial denture. The plain cervical computed tomography showed that the denture was in the hypopharynx. Transoral retrieval of the denture, with the clasp detached from the cervical esophagus, using laryngoscope-guided forceps under local anesthesia.
\end{abstract}

Title: Asymptomatic entrapped denture in the hypopharynx

Shogo Hanai, $\mathrm{MD}^{1}$, Teruhiko Terasawa, $\mathrm{MD}, \mathrm{PhD}^{1}$, Mitsunaga Iwata, $\mathrm{MD}, \mathrm{PhD}^{1}$

1: Department of Emergency and General Internal Medicine, Fujita Health University School of Medicine, Toyoake, Aichi, Japan

Shogo Hanai, MD

Assistant Professor

Department of Emergency and General Internal Medicine

Fujita Health University School of Medicine

470-1192, 1-98, Dengakugakubo, Kutsukake, Toyoake, Aichi, Japan

Telephone: +81-80-1565-4005

Email: honoasa@gmail.com

Key words: aspiration pneumonia, accidental ingestion

All authors have no conflicts of interest directly relevant to the content of this article.

Word counts: 141

A 71-year-old asymptomatic man with cerebral infarction and dementia was brought to our emergency department for ingesting a removable partial denture, as seen incidentally on his follow-up chest x-ray for his aspiration pneumnia provisionally diagnosed three days prior (Figure 1). He could not recall ingesting the denture. We had not auscultated any abnormal respiratory sounds including stridor, wheezing, or crackles. The plain cervical computed tomography showed that the denture was in the hypopharynx (Figure 2,3); a laryngeal endoscopy identified the denture in the upper esophagus, with its metallic clasp entrapped by the postcricoid region. For airway maintenance, tracheostomy was performed, followed by the transoral retrieval of the denture, with the clasp detached from the cervical esophagus, using laryngoscope-guided forceps under local anesthesia. Pressure ulcers were noted but healed spontaneously according to the follow-up laryngeal endoscopy. He was discharged on day 16 . 


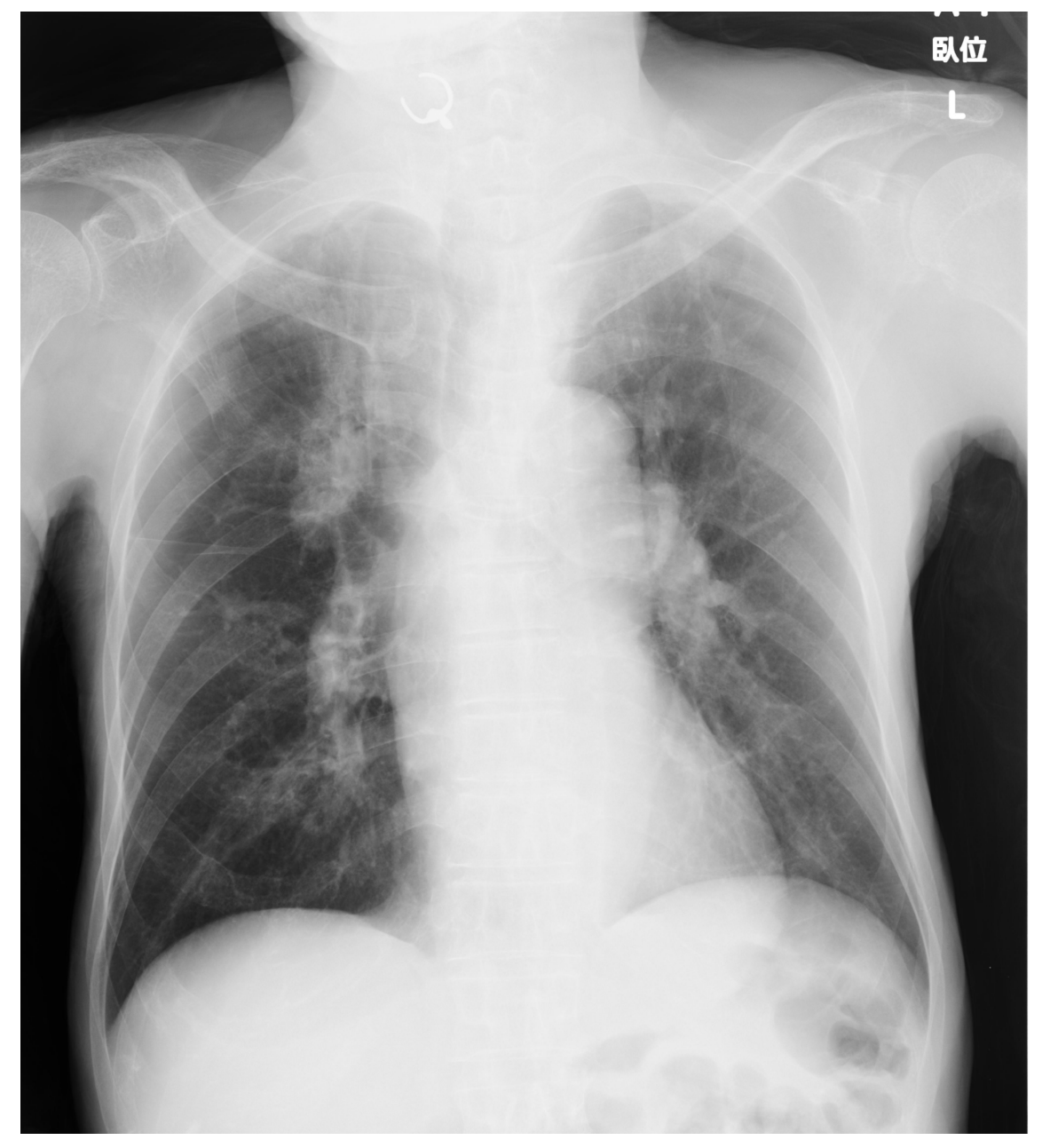




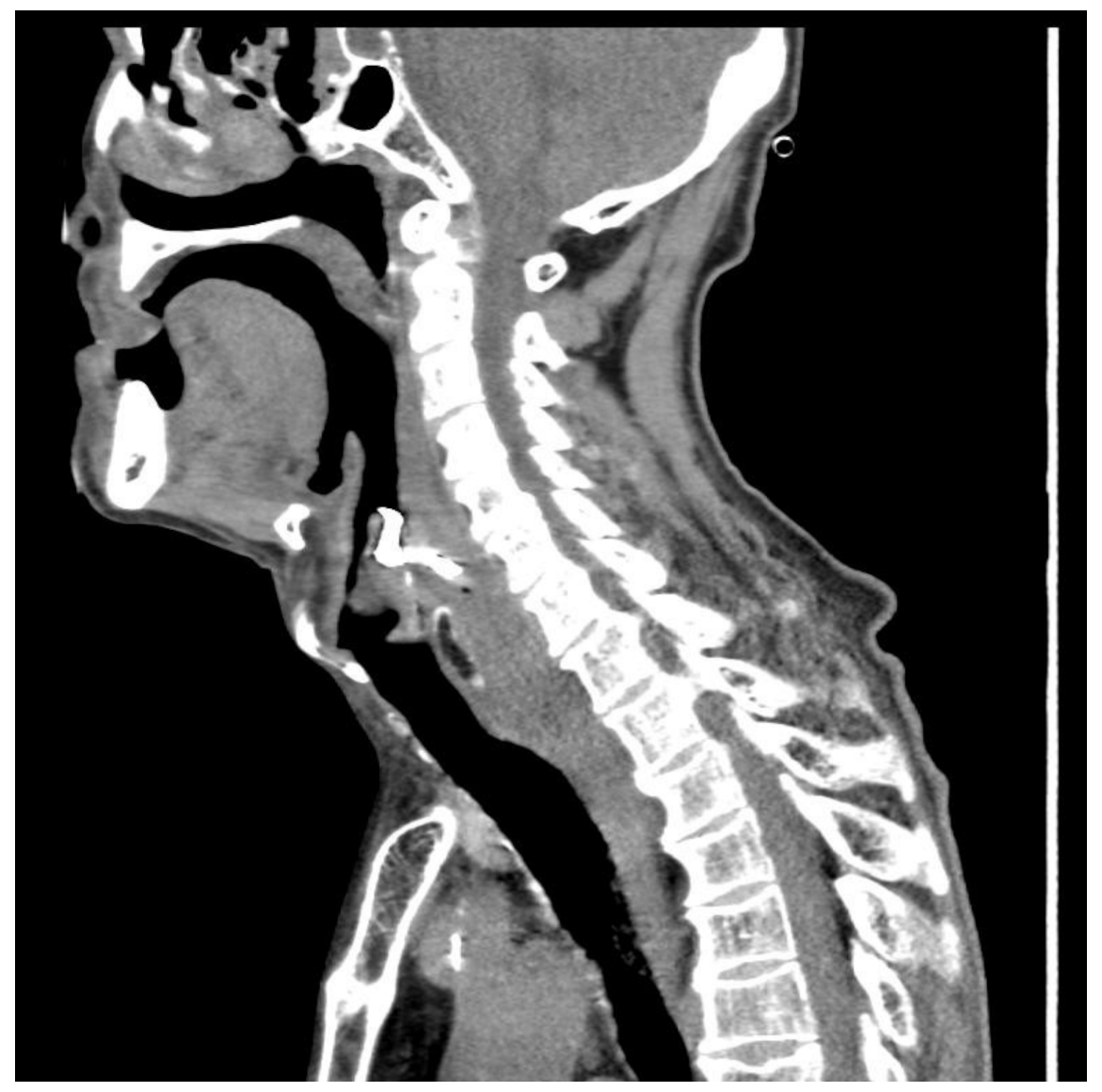




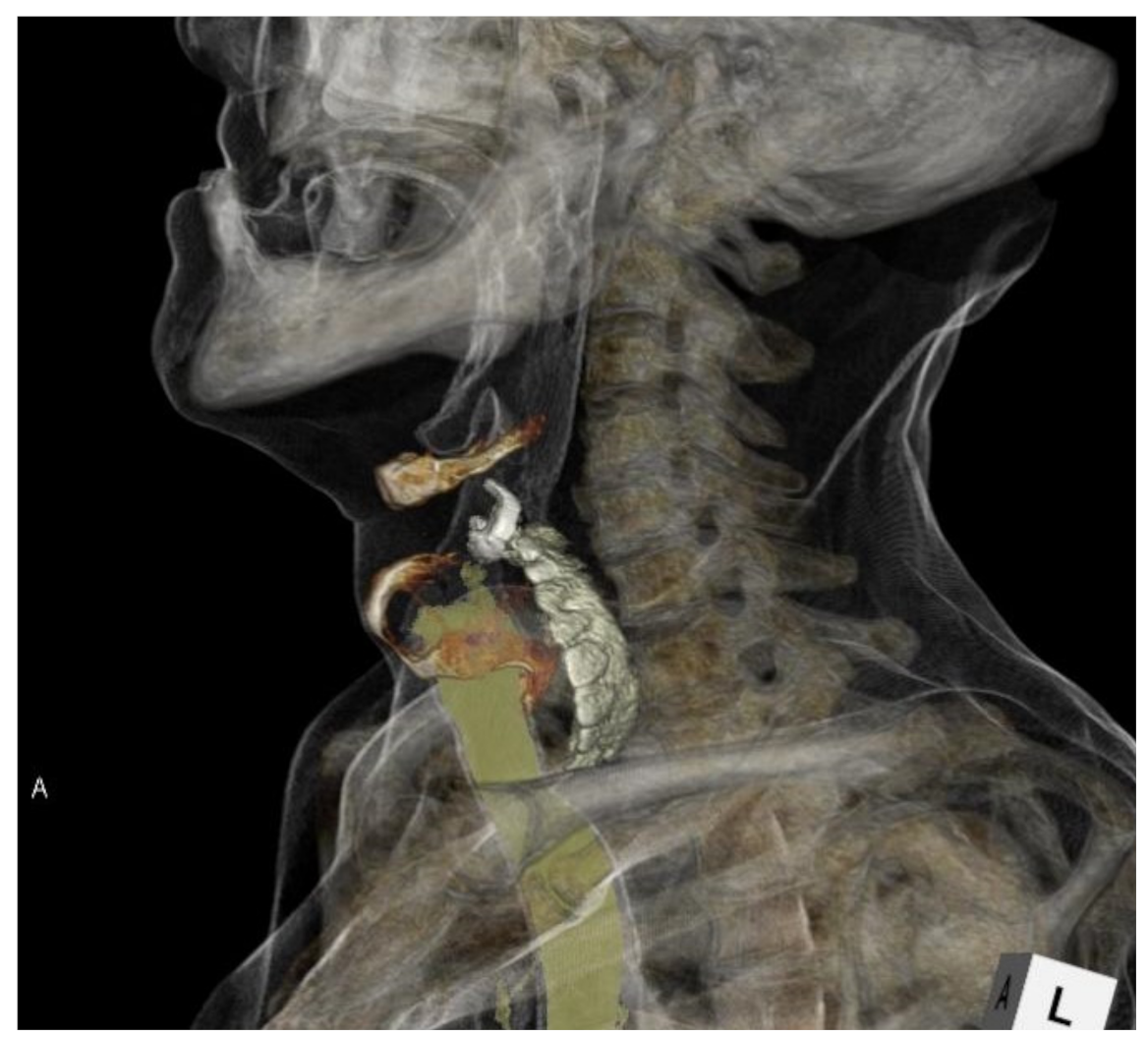

